\title{
Title: Assessment of Systemic Genetic Damage in Pediatric Inflammatory Bowel Disease
}

Authors: Ayesha Baig ${ }^{1}$, Svetlana L. Avlasevich², Dorothea K. Torous², Jeffrey C. Bemis², Lawrence J. Saubermann ${ }^{1}$, David P. Lovell ${ }^{3}$, James T. MacGregor ${ }^{4}$, Stephen D. Dertinger ${ }^{2 *}$

\author{
Affiliations: \\ ${ }^{1}$ University of Rochester Medical Center, Dept. Pediatrics, Rochester, NY \\ ${ }^{2}$ Litron Laboratories, Rochester, New York, USA \\ ${ }^{3}$ St. George's University of London \\ ${ }^{4}$ Toxicology Consulting Services, Bonita Springs, FL
}

*Corresponding author: SDD, Litron Laboratories, 3500 Winton Place, Rochester, NY, 14623; sdertinger@litronlabs.com

Key words: Inflammatory bowel disease, genotoxicity; flow cytometry, chromosomal damage, mutation

Running Title: Genetic Damage in Pediatric IBD

This article has been accepted for publication and undergone full peer review but has not been through the copyediting, typesetting, pagination and proofreading process which may lead to differences between this version and the Version of Record. Please cite this article as doi: 10.1002/em.22403 


\section{Abstract}

The etiology of distal site cancers in inflammatory bowel disease (IBD) is not well understood and requires further study. We investigated whether pediatric IBD patients' blood cells exhibit elevated levels of genomic damage by measuring the frequency of mutant phenotype (CD59-/CD55-) reticulocytes (MUT RET) as a reporter of PIG-A mutation, and the frequency of micronucleated reticulocytes (MN-RET) as an indicator of chromosomal damage. IBD patients ( $n=18$ new onset disease, 46 established disease) were compared to agematched controls (constipation or irritable bowel syndrome patients from the same clinic, $n=30$ ) and young healthy adults age $19-24(n=25)$. IBD patients showed no indication of elevated MUT RET relative to controls (mean \pm std. dev. $=3.1 \pm 2.3 \times 10^{-6}$ versus $3.6 \pm 5.6 \times 10^{-6}$, respectively). In contrast, of 59 IBD patients where \%MN-RET measurements were obtained, 10 exceeded the upper bound $90 \%$ tolerance interval derived from control subjects (i.e., $0.42 \%$ ). Furthermore, each of the 10 IBD patients with elevated MN-RET had established disease $(10 / 42)$, none were new onset $(0 / 17)(p=0.049)$. Interestingly, each of the subjects with increased chromosomal damage was receiving anti-TNF based monotherapy at the time blood was collected (10/10, 100\%), whereas this therapy was less common (20/32, 63\%) among patients that exhibited $\leq 0.42 \%$ MN-RET $(p=0.040)$. The results clearly indicate the need for further work to understand whether the results presented herein are reproducible, and if so, to elucidate the causative factor(s) responsible for elevated MN-RET frequencies in some IBD patients. 


\section{Introduction}

Inflammatory bowel disease (IBD) is characterized by chronic inflammation in the gastrointestinal tract, and comprises Crohn's disease (CD) and ulcerative colitis (UC). Although the clinical presentation of the two subtypes is usually indistinguishable, their histology, localization of inflammation, and disease-specific complications are discrete [Pereira et al., 2015]. Rising rates of pediatric IBD in both developed and developing nations have been reported, primarily due to the increasing incidence of CD [Benchimol et al., 2011].

While the mechanisms underlying the initial development of IBD are not fully understood, it has been suggested that an abnormal immune response against the intestinal microbiota in genetically predisposed individuals exposed to environmental risk factors is responsible [Abraham and Cho, 2009]. Inflammatory cytokine pathways are believed to play a central role in the pathogenesis of IBD, with an imbalance of effector lymphocytes and pro-inflammatory cytokines resulting in chronic inflammation along the gastrointestinal tract [Christophi et al., 2012] and a large increase in reactive oxygen species (ROS) resulting from the localized activity of cytokines, stimulated leukocytes, and activated macrophages.

One important consequence of IBD is an increased risk of gastrointestinal malignancies. According to Jess and colleagues [2012], the risk of colorectal cancer is increased 2.4-fold in patients with UC, with higher risk in males, those of young age at time of diagnosis, and those with extensive colitis. The risk of malignancy begins to increase significantly above that of the general population approximately 8 to 10 years after diagnosis. For example, a meta-analysis conducted by Eaden and colleagues [2001] reported that colorectal cancer risk in UC is $2 \%$ after 10 years, $8 \%$ after 20 years, and $18 \%$ after 30 years of disease. The overall survival is driven primarily by age, comorbidities, and cancer stage at diagnosis [Annese et al., 2015].

There is strong evidence that prolonged chronic inflammation is the principal causative factor in colitis-associated gastrointestinal carcinomas [Axelrad et al., 2016]. As reviewed by Hanahan and Weinberg [2011], local inflammation is considered a hallmark of cancer, and likely 
contributes to neoplastic transformation by enhancing angiogenesis and risk of metastasis as the result of extracellular matrix degradation, and through genetic alterations caused by reactive oxygen and nitrogen species.

While the IBD/cancer link is obvious for gastrointestinal tissues, it is less clear for distal sites. Increased risk of non-melanoma skin cancers, non-Hodgkin lymphoma, acute myeloid leukemia, and urinary tract cancers has been reported, but it is difficult to attribute these to the disease per se versus various immunosuppressive therapies that have historically been used to treat the disease [Axelrad et al., 2016; Jones et al., 2007; Pedersen et al., 2010; Pasternak et al., 2013]. Indeed, the relationship between IBD, hematopoietic cell cancers, and drug therapy has been a concern at least since Hecker et al. [1978] described Hodgkin's disease in conjunction with azathioprine treatment. Since this early report, the use of thiopurines for IBD has consistently been shown to increase the overall risk of cancer, which is reversible upon discontinuation of therapy [Khan et al., 2013].

Thus, the challenge of untangling the etiology of hematological cancers in IBD has been complicated by the historic use of immunomodulating agents that are themselves tumorigenic. Modern treatment approaches that tend to rely more on monotherapies may enable a better understanding of causative factors and their relative contributions. For instance, Lemaitre and colleagues [2017] studied a large cohort of French IBD patients treated from 2009 to 2013 and followed through 2015. In this cohort, 50,405 were treated with thiopurine monotherapy, 30,294 with anti-TNF monotherapy, and 14,229 with a combination of the two. The number of unexposed patients was 123,069. Relative to unexposed patients, Lemaitre and colleagues found that lymphoma risk was elevated among patients treated with thiopurine monotherapy (adjusted hazard ratio of $2.60 ; 95 \% \mathrm{Cl}=1.96-3.44 ; p<0.001$ ), anti-TNF monotherapy $(2.41$; $1.60-3.64 ; p<0.001)$, and combination therapy $(6.11 ; 3.46-10.8 ; p<0.001)$. The authors noted that the elevated hazard ratio observed for the combination therapy may in part be due to the fact that combination therapy tends to be used in the most severe cases. 
The present work was stimulated by experiments conducted by Westbrook and colleagues [2009], who reported evidence of systemic genotoxicity in mouse models of IBD. They reported elevated frequencies of micronucleated erythrocytes in chemical- and immunemediated colitis mouse models, and they also observed 8-oxoguanine- and $\gamma \mathrm{H} 2 \mathrm{AX}$-positive leukocytes. Since genomic damage is recognized as playing a key role in the multistep process of carcinogenesis, this work suggested that IBD itself (at least in several mouse models) may be responsible for some portion of the elevated risk of hematopoietic cancers. More recently, Cao et al. [2020] extended the study of genotoxicity biomarkers from laboratory rodent models to IBD patients in China. They found that adult IBD patients exhibited markedly higher micronucleated lymphocyte frequencies compared to an age-matched healthy adult population. However, since all of those IBD patients had experienced azathioprine-based therapy, it is impossible to determine whether elevated micronucleated lymphocyte frequencies were caused by the disease, thiopurine treatment, or some combination of the two.

We sought to extend these genotoxicity-centric investigations to a pediatric IBD population using two cross-species compatible flow cytometry-based biomarkers that are applicable to human studies: the frequency of mutant phenotype (CD59-/CD55-) reticulocytes (MUT RET) as a reporter of PIG-A gene mutation, and the frequency of micronucleated reticulocytes (MN-RET) as an indicator of chromosomal damage [Torous et al., 2020]. We compared MUT RET and MN-RET data from IBD patients (18 new onset and 46 established) to age-matched controls consisting of 30 constipation or irritable bowel syndrome patients from the same clinic, as well as 25 young healthy adults.

\section{Materials and Methods}

\section{Subject Information}

Operating under IRB-approved protocols, 94 pediatric patients associated with the University of Rochester Medical Center's Department of Pediatrics provided informed consent 
and donated blood sample(s) via venipuncture of the median cubital or cephalic vein at the cubital fossa. Blood was collected into a single $4 \mathrm{~mL} \mathrm{~K}$-EDTA vacutainer tube. These 94 pediatric patients were either diagnosed with IBD, constipation, or irritable bowel syndrome (IBS), and provided blood samples at the University of Rochester Medical Center or an affiliated clinic. Blood samples were transported to Litron in Exakt Paks with $-20^{\circ} \mathrm{C}$ ice packs to keep them cold but not frozen during same day delivery. Blood samples from self-reported healthy young adults $(n=25)$ were purchased from Biological Specialty Company (Colmar, PA). In these cases, 4 or $10 \mathrm{~mL} \mathrm{~K}$-EDTA vials were shipped in Exakt Pak or comparable containers with $-20^{\circ} \mathrm{C}$ ice packs in order to keep them cold, not frozen, during FedEx First Overnight delivery service. Additional information about subject characteristics and the blood samples is provided in Table I. Note that erythrocyte sedimentation rate, C-reactive protein, and Pediatric Crohn's Disease Activity Index [Otley et al., 1999] were measured according to standard clinical practices. The non-standard blood-based assays are described in more detail, below.

\section{PIG-A Assay: Reagents}

Lympholyte®-H was purchased from CedarLane, Burlington, NC. Anti-PE MicroBeads, LS Columns, and QuadroMACS ${ }^{\mathrm{TM}}$ Separator were from Miltenyi Biotec, Bergisch Gladbach, Germany. CountBright ${ }^{\mathrm{TM}}$ Absolute Counting Beads and fetal bovine serum (FBS) were purchased from Invitrogen, Carlsbad, CA. Anticoagulant Solution, balanced salt solution (BSS), Nucleic Acid Dye Solution (a 1000-fold dilution of SYTO® 13 dye in DMSO), anti-human-CD59PE (clone OV9A2), anti-human-CD55-PE (clone 143-30), anti-human-CD45-PE (clone HI30), and anti-human-CD61-PE (clone VI-PL2) were from Prototype Human MutaFlow® Kits (Litron Laboratories, Rochester, NY). Blood Freezing and Blood Thawing Solutions A and B were from MutaFlow B Blood Freezing and Thawing Kits (Litron Laboratories, Rochester, NY).

\section{PIG-A Assay, Sample Processing and Flow Cytometric Analysis}


The methods for preparing blood samples for MUT RET scoring via flow cytometric analysis have been described in detail by Torous et al. [2020]. A brief description follows. Upon receipt, $240 \mu \mathrm{L}$ of whole blood were transferred into kit-supplied Freezing Solution then transferred to a -75 to $-85^{\circ} \mathrm{C}$ freezer. Samples were frozen for at least $48 \mathrm{hr}$ before $P I G-A$ analysis.

On the day of FCM analysis, frozen blood samples were removed from the ultracold freezer and thawed in a $37^{\circ} \mathrm{C}$ water bath. Blood suspensions were brought into contact with kitsupplied Thawing Solution A and then Thawing Solution B, and then layered onto LympholyteH. Following centrifugation, the cells were brought into contact with anti-CD59-PE, anti-CD55PE, anti-CD61-PE, and anti-CD45-PE, and after incubation and centrifugation, cells were mixed with superparamagnetic anti-PE MicroBeads. Following incubation and centrifugation, a small aliquot of each sample was stained with Nucleic Acid Dye Solution that contained CountBright Absolute Counting Beads. These "pre-column" samples were stored on ice until flow cytometric analysis occurred as described below.

The remainder of each sample was applied to a pre-wetted Miltenyi LS Column suspended in a QuadroMACS Separator. Cells found in the eluates (enriched for mutant phenotype cells) were concentrated by centrifugation and finally stained with the same Nucleic Acid Dye Soluton and CountBright Beads used to prepare pre-column samples. These "post-column" samples were stored on ice until flow cytometric analysis occurred as described below.

Samples were analyzed by a flow cytometer equipped with a 488-nm laser (FACSCalibur, BD, San Jose, CA). Nucleic Acid Dye, anti-CD59-PE, anti-CD55-PE, anti-CD45PE, anti-CD61-PE, and Counting Beads fluorescence signals were detected in the FITC, PE, and PerCP-Cy5.5 channels. Calibration of the flow cytometer was accomplished by generating an Instrument Calibration Standard on each day data acquisition occurred. The resulting samples provided sufficient numbers of mutant-mimic cells (erythrocytes that were stained with 
Nucleic Acid Dye but not incubated with antibody solution), and were used for optimizing photomultiplier tube voltages and fluorescence compensation settings. The position of the mutant mimics also provided a rational approach for defining the vertical demarcation line used to distinguish mutant phenotype cells from wild-type cells.

After photomultiplier tube voltages and electronic compensation settings were defined with the Instrument Calibration Standard, pre- and post-column samples were moved into standard flow cytometer tubes and analyzed within $2 \mathrm{hr}$. Data acquisition continued until a specified time: 1 min for every pre-column sample, and 3 min.

\section{Micronucleus Assay: Reagents}

Absolute methanol and heat-inactivated fetal bovine serum (FBS; cat. no. 89510-186) were from VWR, Radnor, PA. Reagents used for flow cytometric MN-RET scoring (Anticoagulant Solution, Buffer Solution, DNA Stain, Anti-CD71-FITC and Anti-CD61-PE Antibodies, RNase Solution, and Malaria Biostandards) were from Prototype In Vivo MicroFlow® PLUS-H Kits, Litron Laboratories, Rochester, NY. Anti-FITC MicroBeads, MS Columns, and an OctoMACS ${ }^{\mathrm{TM}}$ Separator were from Miltenyi Biotec, Bergisch Gladbach, Germany.

\section{Micronucleus Assay: Sample Processing and Flow Cytometric Analysis}

The methods for preparing blood samples for MN-RET scoring via flow cytometric analysis have been described in detail [Torous et al., 2020]. A brief description follows. Blood samples were fixed with ultracold methanol and stored in a -75 to $-85^{\circ} \mathrm{C}$ freezer. On the day of analysis, samples were washed out of fixative, incubated with anti-CD71-FITC and anti-CD61PE, and after a wash step they were brought into contact with superparamagnetic anti-FITC MicroBeads. Samples were divided into two fractions: "pre-column" and "post-column". For pre- 
column samples, a $20 \mu \mathrm{L}$ aliquot of each cell suspension was added to Buffer Solution with RNase and DNA Stain. Flow cytometric analysis occurred as described below.

The remainder (majority) of each sample was applied to a pre-wetted Miltenyi MS Column suspended in an OctoMACS Separator. Column-bound cells (highly enriched for CD71positive reticulocytes) were flushed out and then concentrated via centrifugation. The cells were stained with the same DNA Stain, RNase and Buffer Solution used for the pre-column samples.

Flow cytometer instrumentation and acquisition/analysis software parameters were calibrated based on the fluorescence of a biological standard-kit supplied malaria-infected rat blood (methanol fixed). As described previously, this sample guided photomultiplier tube voltages and compensation settings to resolve parasitized reticulocytes and also guided the position of the micronucleus scoring region [Tometsko et al., 1994; Dertinger et al., 2000]. Data acquisition and analyses were performed using a FACSCalibur flow cytometer providing $488 \mathrm{~nm}$ excitation, running CellQuest ${ }^{\mathrm{TM}}$ Pro software v5.2.

Once photomultiplier tube voltages and compensation settings were configured with the malaria biostandard, the pre- and post-column paired samples were analyzed. Pre-column sample data acquisition occurred until 300,000 total erythrocytes were collected. While the precolumn sample was being analyzed, $300 \mu \mathrm{L}$ of the same working DNA stain was added to each post-column tube and the entire volume was transferred into a standard flow cytometer tube. Each sample was analyzed so that at least 10,000, but preferably 20,000 CD71-positive RET were analyzed. Note that we do not report \%MN-RET for 14 of 143 samples $(9.8 \%$ of total) because pre-column RET was $<0.02 \%$ and we were unable to acquire at least 10,000 RET.

\section{Statistical Analyses}

The formulas used to calculate MUT RET frequencies based on pre- and postimmunomagnetic column data have been published [Dertinger et al., 2012] and are described in MutaFlow Kit manuals (www.litronlabs.com). The incidence of MN-RET is expressed as 
frequency percent, while the incidence of MUT RET is expressed as number per $10^{6}$ cells. For statistical evaluations, MUT RET and MN-RET frequencies were log(10) transformed.

A one-sided (upper bound) TI was calculated for the MUT RET and MN-RET biomarkers based on data from relatively healthy young volunteers: pediatric constipation and IBS patients, and young healthy adults. A TI is a statistic interval which, for a specific confidence level, a specified proportion of a sampled population falls within [Vardeman, 1992]. JMP software's distribution platform was used for these analyses and coverage was set to $90 \%$, alpha $=0.05$ (JMP v12.0.1, SAS Institute Inc., Cary, NC). Thus, we used the resulting upper bound TIs to provide cutoff values indicative of elevated MUT RET and MN-RET values.

JMP software's fit $Y$ by $X$ platform was used to perform a two-sided t-test (assuming unequal variances; alpha $=0.05$ ) comparing MN-RET frequencies for IBD patients that were categorized as "new onset" versus "established disease". For the purpose of this presentation, new onset was defined as patients that had histological diagnosis of IBD within the past $0-12$ months of blood collection for this study but had either received no drug treatment or were in the induction phase of receiving biologics (i.e., less than 3 infusions). Established disease was defined as patients that had histological diagnosis of IBD and were on a scheduled regimen for receiving biologics or else small molecule drug therapy.

The $\mathrm{Y}$ by $\mathrm{X}$ platform was also used to conduct two-sided Fisher's exact tests, alpha = 0.05. One test concerned the association between MN-RET frequency, elevated or not, relative to IBD category, established or new onset. A second test investigated the association between MN-RET frequency, elevated or not, relative to current drug therapy, infliximab or not.

Finally, the $\mathrm{Y}$ by $\mathrm{X}$ platform was used to conduct two-sided t-tests (assuming unequal variances; alpha $=0.05)$ to compare CD71-positive reticulocyte frequencies in pediatric non-IBD patients, new onset IBD patients, and established IBD patients.

\section{Results}




\section{Mutant Reticulocytes}

MUT RET values for pediatric constipation and IBS patients, as well as healthy young adults, are shown in Figure 1, left panel. Based on these results, an upper bound 90\% TI was calculated $\left(10 \times 10^{-6}\right.$, shown as a dashed red line). We took MUT RET values greater than this to be indicative of an elevated level of gene mutation. MUT RET values for pediatric IBD patients are shown in Figure 1, right panel. All but one subject exhibited MUT RET values that were below the upper bound TI. The single patient with an elevated frequency of mutant phenotype cells was an exceptionally high outlier, a $13 \mathrm{yr}$ old female established IBD patient with a MUT RET value of $500 \times 10^{-6}$ (off scale). There was nothing otherwise remarkable about this patient. Aside from this one extreme outlier, IBD patients showed no indication of elevated MUT RET relative to controls (mean \pm std. dev. $=3.1 \pm 2.3 \times 10^{-6}$ versus $3.6 \pm 5.6 \times 10^{-6}$, respectively). These data suggest MUT RET frequency is not markedly affected by IBD, or by the therapies used to treat these particular IBD patients. That being said, it is important to note that aside from infliximab, no other drugs were sufficiently represented to adequately assess their genotoxic potential (see Table II).

\section{Micronucleated Reticulocytes}

$\% \mathrm{MN}-\mathrm{RET}$ values for pediatric constipation and IBS patients, as well as healthy young adults, are shown in Figure 2, left panel. Based on these results, an upper bound 90\% TI was calculated $(0.42 \%$, shown as a dashed red line). We took MN-RET values greater than this to be indicative of an elevated level of chromosomal damage. MN-RET values for pediatric IBD patients are shown in Figure 2, right panel. Of 59 IBD patients, 10 showed elevated MN-RET values. All 10 were from the 42 established disease patients, and 0 of 17 were from the new onset disease subpopulation. A Fisher's exact test that considered the association between $\mathrm{MN}$ RET category (elevated or not) relative to IBD category (new onset or established) resulted in a $p$ value of 0.049 . A highly significant two-tailed t-test result $(p<0.001)$ strengthens the 
conclusion that \%MN-RET were significantly elevated in established IBD patients compared to new onset: mean \pm std. dev. $=0.31 \pm 0.32 \%$ versus $0.13 \pm 0.09 \%$, respectively.

Besides this association between elevated MN-RET frequency and established disease, other potential explanatory factors including age, time since diagnosis, and indices of inflammation/disease severity are described in Table II. No obvious associations are evident from this relatively small study, with one notable exception that all of the subjects with increased chromosomal damage were undergoing anti-TNF monotherapy (infliximab) at the time blood was collected (10/10, 100\%). Infliximab therapy was less common among patients that exhibited $\leq 0.42 \%$ MN-RET (20/32, 63\%). See Figure 3. A Fisher's exact test that considered the association between MN-RET category (elevated or not) relative to infliximab therapy (yes or no) resulted in a $p$ value of 0.040 .

\section{Incidental Finding: CD71-Positive Reticulocytes}

The flow cytometric method of scoring MUT RET frequencies simultaneously quantifies the percentage of immature reticulocytes, i.e., the CD71+ fraction, relative to the total number of erythrocytes. An incidental finding is that this biomarker of erythropoiesis function was elevated in the new onset IBD patients relative to the two other pediatric populations-both the constipation/IBS patients ("non-IBD”, $p<0.001)$ and the established IBD patients $(p<0.001)$. See Figure 4.

\section{Discussion}

We found no indication that the pediatric IBD patients studied exhibited elevated MUT RET frequencies relative to controls. Recently, Cao et al. [2020] reported a slight increase in mutant phenotype erythrocytes in Chinese IBD patients. Two differences between these studies are noteworthy, and one is related to methodology. Since our scoring approach was based on reticulocytes rather than erythrocytes, it more effectively reduces the influence that complement- 
mediated lysis can have on mutant cell frequency measurements [Ware et al., 1995; Dertinger et al., 2015; Torous et al., 2020]. Second, the patients studied by Cao and colleagues were all treated with azathioprine, a recognized genotoxic agent [Dertinger et al., 2012].

In contrast to MUT RET, we observed elevated frequencies of the chromosomal damage biomarker, MN-RET. This is consistent with Cao and colleagues who also reported significantly elevated frequencies of micronucleated cells (in their case, lymphocytes). However, the same qualification noted above applies—-the patients in the Cao et al. study were all treated with azathioprine, so direct comparisons across these studies are not possible.

The elevated MN-RET frequencies reported herein are consistent with Westbrook et al. [2009] and Trivedi and Jena [2012], who showed mouse models of IBD exhibit significantly elevated micronucleated cell frequencies. However, it should be noted that those investigators observed increased micronucleus frequencies within days of initiating dextran sulfate sodium treatments, whereas our patients with elevated \%MN-RET were entirely comprised of patients with long-standing/established disease. In any event, the mouse model data are important, because they were observed in the absence of therapeutic drugs, suggesting IBD itself may be responsible for an appreciable level of hematopoietic cell genotoxicity.

Our finding that elevated MN-RET frequencies were associated with infliximab therapy was somewhat surprising. The US FDA-approved label for infliximab indicates that genotoxicity was not apparent in pre-clinical models [Remicade ${ }^{\circledR}$ label, 2013]. It is also important to point out that together with other anti-TNF therapies, infliximab has revolutionized the treatment of IBD [Kierkus et al., 2015]. Therefore, any possible deleterious effects from infliximab must be weighed carefully against the often profound benefits to patients' health and quality of life. Finally, the association we observed between elevated MN-RET frequencies and infliximab therapy is just that-an association. It is not a proven causative link. It is possible that the significant association we described may be related to as-yet unidentified aspect(s) of the disease itself, with time since diagnosis representing an underlying component. 
When measuring \%MN-RET via flow cytometry, the frequency of transferrin receptor (CD71) positive reticulocytes among total erythrocytes is also recorded. CD71-positive reticulocytes are a subpopulation of RNA-positive reticulocytes, i.e., they are the most immature fraction [Serke and Huhn, 1992]. An incidental finding of our study is that the frequency of CD71-positive reticulocytes among total erythrocytes is elevated in many new onset IBD patients as compared to non-IBD and established IBD patients. These elevated levels may reflect increased erythropoiesis function, and the clinical value of this metric warrants further study.

In conclusion, the relatively small size of this study limits the certainty of the statistical associations we have observed and makes it premature to reach definitive conclusions about the causative factors responsible for the observed elevated levels of chromosomal damage. However, the results clearly indicate the need for further work to assess whether the results presented herein are reproducible, and if so, to elucidate causative factor(s) of elevated MNRET values and of hematopoietic malignancies in IBD patients.

\section{AUTHOR CONTRIBUTIONS}

S.D.D., J.C.B., L.J.S., D.P.L., and J.T.M. designed the study; A.B. and L.J.S. oversaw IRB approval; A.B. recruited and consented GI-clinic volunteers; D.K.T. oversaw the purchase and receipt of healthy young adult blood samples; S.L.A. and D.K.T. processed blood samples and performed flow cytometric analyses; S.D.D. and A.B. performed statistical analyses under the direction of D.P.L.; A.B. and S.D.D. wrote the initial draft of manuscript that was substantially edited and finally approved by all co-authors.

\section{CONFLICT OF INTEREST STATEMENT}


S.L.A., D.K.T., J.C.B and S.D.D are employed by Litron Laboratories. Litron owns patents covering the flow cytometry-based assays described in this manuscript and plans to sell commercial kits based on these procedures: J.T.M. serves as a paid consultant to Litron.

\section{ACKNOWLEDGMENTS}

This work was funded by a grant from the National Institute of Health/National Institute of Environmental Health Sciences (NIEHS; grant number R44ES028163). The contents are solely the responsibility of the authors, and do not necessarily represent the official views of the NIEHS.

\section{Figure Legends}

Figure 1. Individual subjects' mutant reticulocyte frequencies are graphed against their age in years. The left panel shows relatively heathy controls, where red diamonds indicate pediatric constipation patients, green circles indicate pediatric irritable bowel syndrome patients, and black diamonds indicate self-reported healthy young adults. The red dashed line marks the mutant reticulocyte frequency upper bound $90 \%$ tolerance interval, alpha $=0.05$ (i.e., $10 \times 10^{-6}$ ). Note that one 19-year old male subject's mutant reticulocyte frequency is included in the tolerance interval calculation, but the data point is off-scale (i.e., $40.7 \times 10^{-6}$ ). The right panel shows pediatric inflammatory bowel disease patients, where unfilled blue squares indicate new onset patients and filled blue squares indicate established patients. The upper bound tolerance interval is again indicated by a red dashed line.

Figure 2. Individual subjects' micronucleated reticulocyte frequencies (MN-RET) are graphed against their age in years. The left panel shows relatively heathy controls, where red diamonds indicate pediatric constipation patients, green circles indicate pediatric irritable bowel syndrome patients, and black diamonds indicate self-reported healthy young adults. The red dashed line 
marks the MN-RET frequency upper bound $90 \%$ tolerance interval, alpha $=0.05$ (i.e., $0.42 \%$ ). The right panel shows pediatric inflammatory bowel disease patients, where unfilled blue squares indicate new onset patients and filled blue squares indicate established patients. The upper bound tolerance interval is again indicated by a red dashed line.

Figure 3. Individual inflammatory bowel disease patient's micronucleated reticulocyte frequencies (MN-RET) are graphed according to their disease subtype (new onset versus established disease) and current treatment: infliximab, yes $(\mathrm{Y})$ or no $(\mathrm{N})$. The red dashed line marks the MN-RET frequency upper bound $90 \%$ tolerance interval, alpha $=0.05$ (i.e., $0.42 \%$ ). As shown here, patients with MN-RET values above the $0.42 \%$ were found exclusively in established disease patients undergoing infliximab therapy.

Figure 4. Individual subject's CD71+ reticulocyte frequencies are graphed according to their disease subtype, where red diamonds indicate pediatric constipation patients, green circles indicate pediatric irritable bowel syndrome patients, unfilled blue squares indicate new onset inflammatory bowel disease patients, and filled blue squares indicate established inflammatory bowel disease patients. The $p$ values correspond to two-sided t-test results, and indicate that new onset inflammatory bowel disease patients exhibit elevated levels of the erythropoiesis biomarker CD71+ reticulocyte frequency. 


\section{REFERENCES}

Abraham, C. and Cho, J.H. (2009) Inflammatory bowel disease. New England Journal of Medicine, 361:2066-2078.

Annese, V., Beaugerie, L., Egan, L., et al. (2015) European evidence-based consensus: inflammatory bowel disease and malignancies. J. Crohns Colitis, 9:945-965.

Axelrad, J.E., Lichtiger, S. and Yajnik, V. (2016) Inflammatory bowel disease and cancer: The role of inflammation, immunosuppression, and cancer treatment. World J. Gastroenterol., 22:4794-4801.

Benchimol, E.I., Fortinsky, K.J., Gozdyra, P., Van den Heuvel, M., Van Limbergen, J. and Griffiths, A.M. (2011) Epidemiology of pediatric inflammatory bowel disease: A systematic review of international trends. Inflammatory Bowel Diseases, 17(1):423-439.

Cao, Y., Wang, X., Liu, W., Feng, N., Xi, J., You, X., Chen, R., Zhang, X., Liu, Z. and Luan, Y. (2020) The potential application of human PIG-A assay on azathioprine treated inflammatory bowel disease patients. Environmental and Molecular Mutagenesis, 61:456464.

Christophi, G.P., Rong, R., Holtzapple, P.G., et al. (2012) Immune markers and differential signaling networks in ulcerative colitis and Crohn's disease. Inflammatory Bowel Diseases, 18:2342-2356.

Dertinger, S.D., Torous, D.K., Hall, N.E., Tomesko, C.R. and Gasiewicz, T.A. (2000) Malariainfected erythrocytes serve as biological standards to ensure reliable and consistent scoring of micronucleated erythrocytes by flow cytometry. Mutation Research, 464(2)195200.

Dertinger, S.D., Phonethepswath, S., Avlasevich, S.L., Torous, D.K., Mereness, J., Bryce, S.M., Bemis, J.C., Bell, S., Weller, P. and MacGregor, J.T. (2012) Efficient monitoring of in vivo Pig-a gene mutation and chromosomal damage: Summary of 7 published studies and results from 11 new reference compounds. Toxicological Sciences, 130:328-348. 
Dertinger, S.D., Avlasevich, S.L., Bemis, J.C., Chen, Y. and MacGregor, J.T. (2015) Human erythrocyte PIG-A assay: An easily monitored index of gene mutation requiring low volume blood samples. Environmental and Molecular Mutagenesis, 56:366-377.

Eaden, J.A., Abrams, K.R. and Mayberry, J.F. (2001) The risk of colorectal cancer in ulcerative colitis: a meta-analysis. Gut, 48:526-535.

Hanahan, D. and Weinberg, R.A. (2011) Hallmarks of cancer: the next generation. Cell, 144:646-674.

Hecker, R., Sheers, R. and Thomas, D. (1978) Hodgkin's disease as a complication of Crohn's disease. Med. J. Aust., 2(13):603.

Jess, T., Rungoe, C. and Peyrin-Biroulet, L. (2012) Risk of colorectal cancer in patients with ulcerative colitis: a meta-analysis of population-based cohort studies. Clin. Gastroenterol. Hepatol., 10:639-645.

Jones, J.L. and Loftus, E.V. Jr. (2007) Lymphoma risk in inflammatory bowel disease: Is it the disease or its treatment? Inflammatory Bowel Diseases, 13:1299-1307.

Khan, N., Abbas, A.M. Lichtenstein, G.R., Loftus, E.V. Jr. and Bazzano, L.A. (2013) Risk of lymphoma in patients with ulcerative colitis treated with thiopurines: a nationwide retrospective cohort study. Gastroenterology, 145(5)1007-1015.

Kierkus, J., Szymanska, E., Oracz, G., Wiernicka, A. and Dadalski, M. (2015) Profile of infliximab in the treatment of pediatric Crohn's disease. Pediatric Health, Medicine and Therapeutics, 6:79-85.

Lemaitre, M., Kirchgesner, J., Rudnichi, A., Carrat, F., Zureik, M., Carbonnel, F. and Dray-Spira, R. (2017) Association between use of thiopurines or tumor necrosis factor antagonists alone or in combination and risk of lymphoma in patients with inflammatory bowel disease. JAMA, 318(17)1679-1686. 
Otley, A., Loonen, H., Parekh, N., Corey, M., Sherman, P.M. and Griffiths, A.M. (1999) Assessing activity of pediatric Crohn's disease. Which Index to Use? Gastroenterology, 116:527-531.

Pasternak, B., Svanström, H., Schmiegelow, K, Jess, T. and Hviid, A. (2013) Use of azathioprine and the risk of cancer in inflammatory bowel disease. Am. J. Epidemiol., 177:1296-1305.

Pedersen, N., Duricova, D., Elkjaer, M., Gamborg, M., Munkholm, P. and Jess, T. (2010) Risk of extra-intestinal cancer in inflammatory bowel disease: Meta-analysis of population-based cohort studies. Am. J. Gastroenterol., 105:1480-1487.

Pereira, C., Grácio, D., Teixeira, J.P. and Magro, F. (2015) Oxidative Stress and DNA Damage: Implications in Inflammatory Bowel Disease. Inflammatory Bowel Diseases, 21(10):24032417.

Remicade ${ }^{\circ}$ U.S. Food and Drug Administration approved label (2013), available at: https://www.accessdata.fda.gov/drugsatfda docs/label/2013/103772s5359lbl.pdf, successfully accessed May 16, 2020.

Serke, S. and Huhn, D. (1992) Identification of CD71 (transferrin receptor) expressing erythrocytes by multiparameter-flow-cytometry (MP-FCM): correlation to the quantitation of reticulocytes as determined by conventional microscopy and by MP-FCM using a RNAstaining dye. British Journal of Haematology, 81:432-439.

Tometsko, A.M, Torous, D.K. and Dertinger, S.D. (1994) Analysis of micronucleated cells by flow cytometry. 1. Achieving high resolution with a malaria model. Mutation Research, 292(2):129-135.

Torous, D.K., Avlasevich, S.L., Khattab, M.G., Baig, A., Saubermann, L.J., Chen, Y., Bemis, J.C., Lovell, D.P., Walker, V.E., MacGregor, J.T. and Dertinger, S.D. (2020) Human blood PIG-A mutation and micronucleated reticulocyte flow cytometric assays: Method 
optimization and evaluation of intra- and inter-subject variation. Currently under review at Environmental and Molecular Mutagenesis.

Trivedi, P.P. and Jena, G.B. (2012) Dextran sulfate sodium-induced ulcerative colitis leads to increased hematopoiesis and induces both local as well as systemic genotoxicity in mice. Mutation Research, 744:172-183.

Vardeman, S.B. (1992) What about the other invervals? The American Statistician, 46(3):193197.

Ware, R.E., Rosse, W.F. and Hall, S.E. (1995) Immunophenotypic analysis of reticulocytes in paroxysmal nocturnal hemoglobinuria. Blood, 86(4):1586-1589.

Westbrook, A.M., Wei, B., Braun, J. and Schiestl, R.H. (2009) Intestinal mucosal inflammation leads to systemic genotoxicity in mice. Cancer Res., 69(11):4827-4834. 
Figure 2.

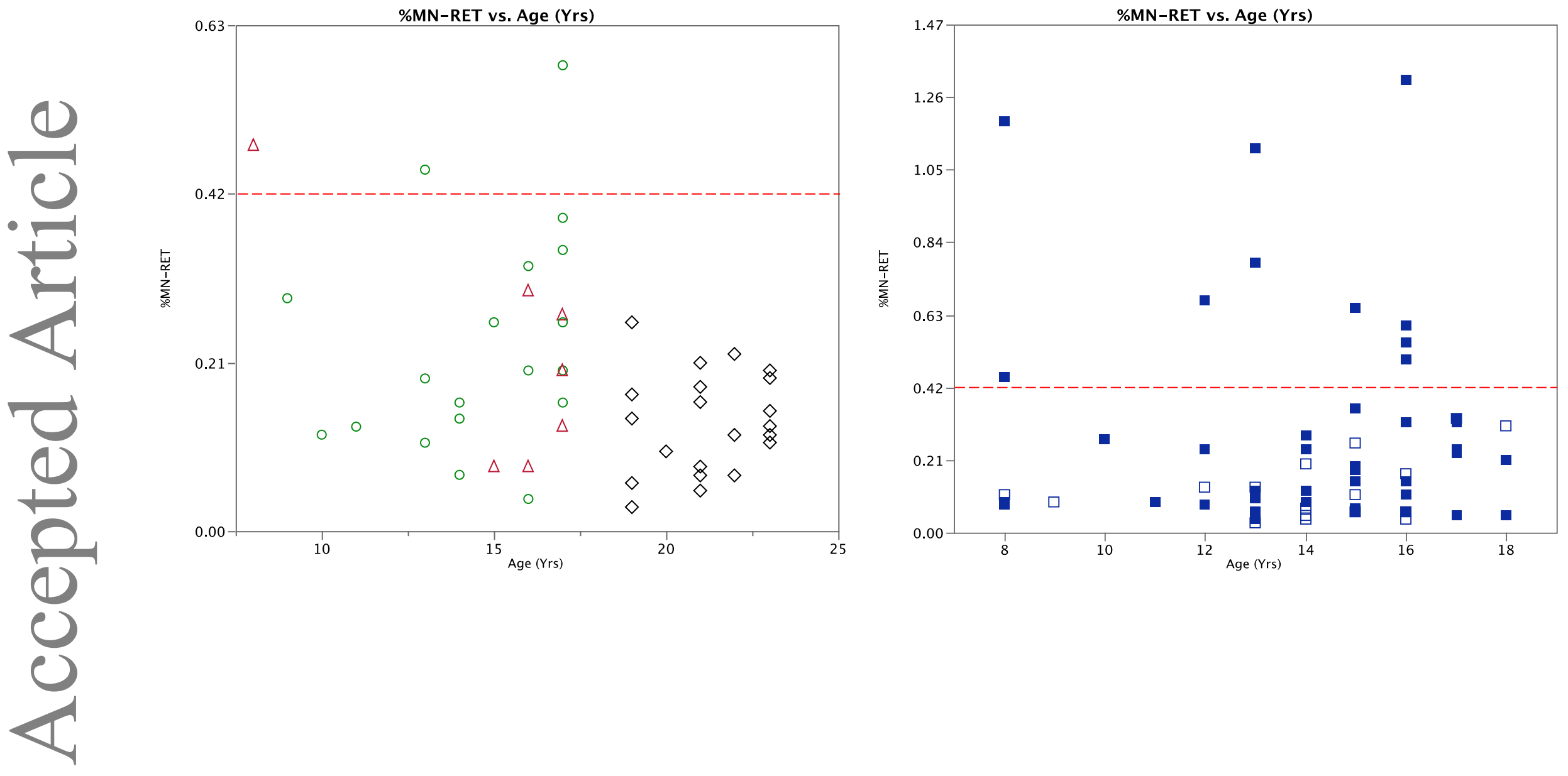


Figure 3.

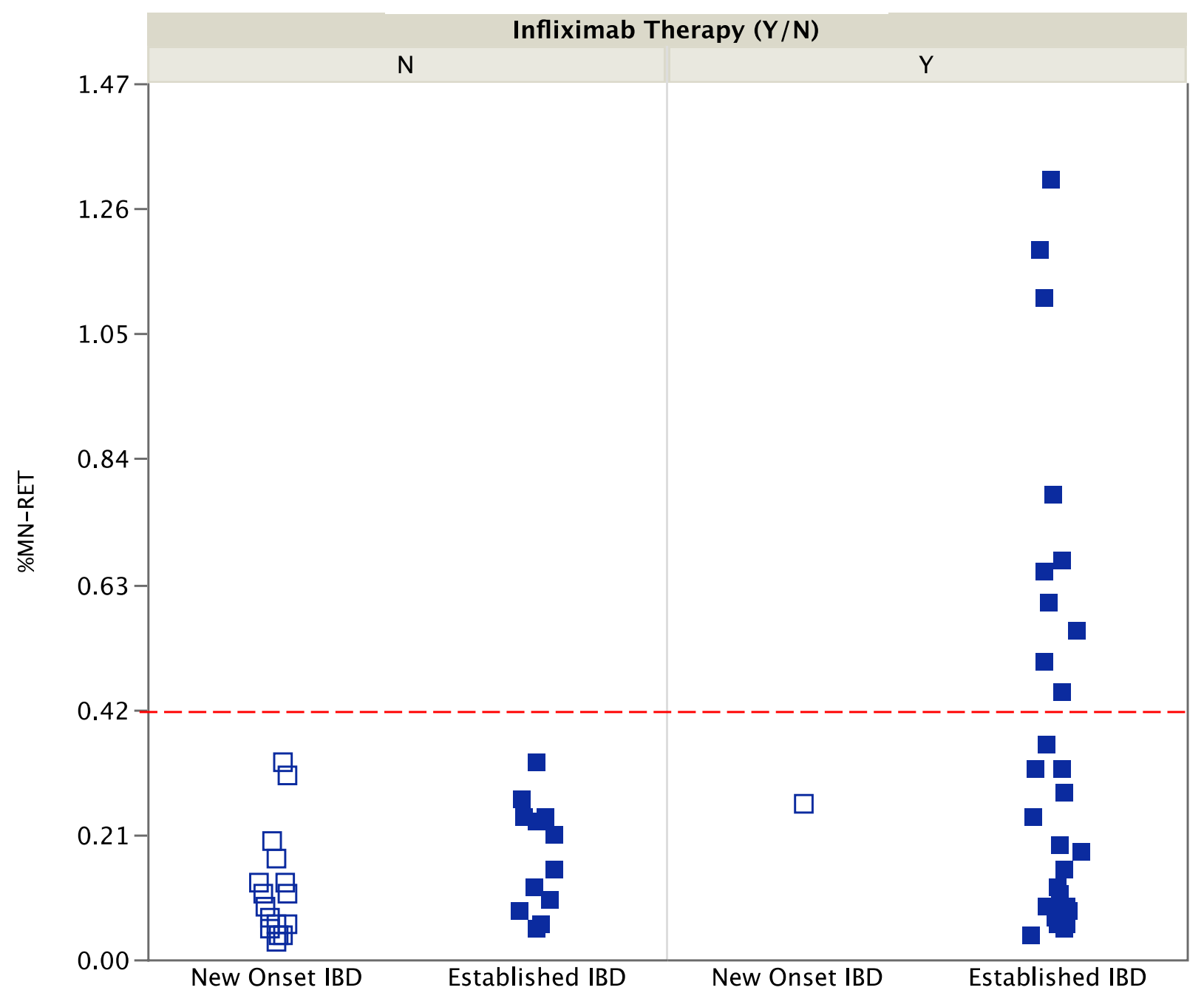


Figure 4.

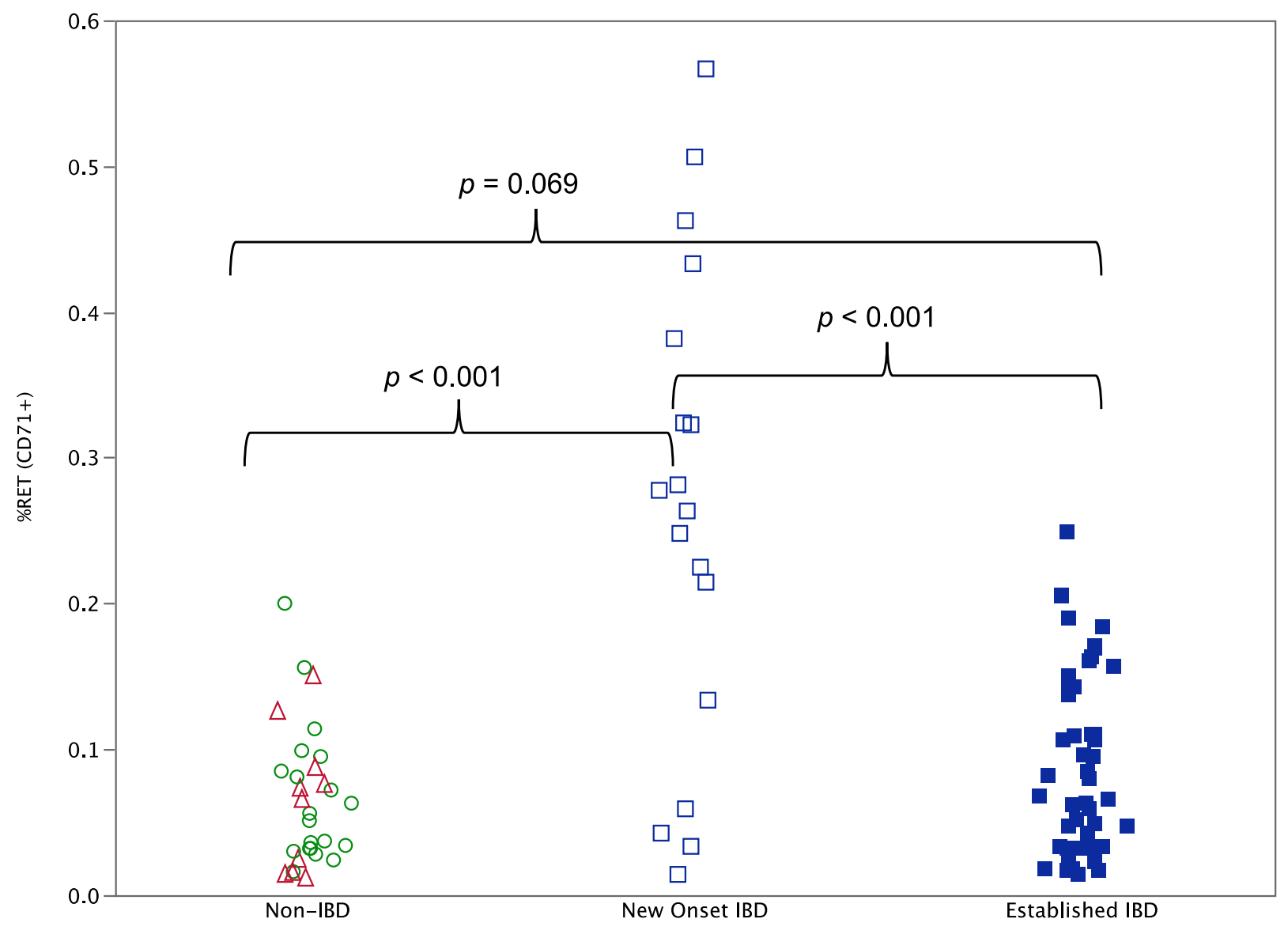


Table I. Subject Characteristics.

\begin{tabular}{|c|c|c|c|c|c|c|c|}
\hline Diagnosis & Source & $\mathbf{N}$ & $\begin{array}{c}\text { No. } \\
\text { Males, } \\
\text { Females }\end{array}$ & $\begin{array}{c}\text { Age Range } \\
\text { (Yrs) }\end{array}$ & $\begin{array}{l}\text { Avg. Age } \\
\pm \text { Std. Dev. } \\
\text { (Yrs) }\end{array}$ & $\begin{array}{c}\text { IBD } \\
\text { Subtype }\end{array}$ & Misc. Notes \\
\hline $\begin{array}{l}\text { Healthy Young } \\
\text { Adults }\end{array}$ & $\begin{array}{l}\text { Biological } \\
\text { Specialty } \\
\text { Company }\end{array}$ & 25 & 18,7 & $19-24$ & $21.2 \pm 1.5$ & NA & \\
\hline $\begin{array}{l}\text { Pediatric } \\
\text { Gastroenterology } \\
\text { Clinic, Non-IBD }\end{array}$ & $\begin{array}{l}\text { URMC, Dept. } \\
\text { Pediatrics }\end{array}$ & 30 & 11,19 & $8-17$ & $14.3 \pm 3.0$ & NA & $\begin{array}{l}\mathrm{N}=20 \text { IBS, } 10 \\
\text { constipation }\end{array}$ \\
\hline $\begin{array}{l}\text { Pediatric } \\
\text { Gastroenterology } \\
\text { Clinic, IBD }\end{array}$ & $\begin{array}{l}\text { URMC, Dept. } \\
\text { Pediatrics }\end{array}$ & 64 & 36,28 & $8-18$ & $14.0 \pm 2.7$ & $\begin{array}{c}\mathrm{N}=54 \\
\mathrm{CD}, 8 \mathrm{UC}, \\
2 \mathrm{IC}\end{array}$ & $\begin{array}{l}\mathrm{N}=18 \mathrm{New} \\
\text { Onset IBD, } 46 \\
\text { Established IBD }\end{array}$ \\
\hline
\end{tabular}

Abbreviations: IBD = inflammatory bowel disease; IBS = irritable bowel syndrome; CD = Crohn's disease; UC = Ulcerative colitis, IC $=$ Indeterminant colitis. 
Table II. Characteristics of New vs. Established Inflammatory Bowel Disease Patients in Relation to \%MN-RET.

\begin{tabular}{|c|c|c|c|c|c|c|c|c|c|c|c|}
\hline Diagnosis & $\begin{array}{l}\text { \%MN- } \\
\text { RET }\end{array}$ & $\begin{array}{c}\% \\
\text { Subjects }\end{array}$ & $\begin{array}{c}\text { No. } \\
\text { Males, } \\
\text { Females }\end{array}$ & $\begin{array}{c}\text { Avg. Age } \pm \\
\text { Std. Dev. } \\
\text { (Yrs) }\end{array}$ & $\begin{array}{c}\text { Avg. Yrs } \\
\text { Since } \\
\text { Diagnosis } \pm \\
\text { Std. Dev. }\end{array}$ & $\mathrm{PCDAl}^{\mathrm{a}}$ & $\begin{array}{c}\text { SED }^{\mathrm{b}} \pm \\
\text { Std. Dev. }\end{array}$ & $\begin{array}{l}\text { Avg. C- } \\
\text { Reactive } \\
\text { Protein } \pm \\
\text { Std. Dev. }\end{array}$ & $\begin{array}{l}\text { Infliximab } \\
\text { Treatment }\end{array}$ & $\begin{array}{c}\text { Small } \\
\text { Molecule } \\
\text { Drug }^{c} \\
\text { Treatment }\end{array}$ & $\begin{array}{c}\text { Other } \\
\text { Biologics }^{d} \\
\text { Treatment }\end{array}$ \\
\hline \multirow{2}{*}{$\begin{array}{l}\text { New Onset } \\
\text { IBD }\end{array}$} & $\leq 0.42 \%$ & $\begin{array}{c}100 \% \\
(17 / 17)\end{array}$ & 10,7 & $14.2 \pm 2.7$ & $0.3 \pm 0.5$ & $31 \pm 20$ & $27 \pm 29$ & $19.5 \pm 19.1$ & $6 \%(1 / 17)^{\mathrm{e}}$ & $6 \%(1 / 17)$ & $0 \%$ \\
\hline & $>0.42 \%$ & $\begin{array}{c}0 \% \\
(0 / 17)\end{array}$ & $N A^{f}$ & NA & NA & NA & NA & NA & NA & NA & NA \\
\hline \multirow{2}{*}{$\begin{array}{l}\text { Established } \\
\text { IBD }\end{array}$} & $\leq 0.42 \%$ & $\begin{array}{c}76 \% \\
(32 / 42)\end{array}$ & 18,14 & $14.2 \pm 2.6$ & $3.7 \pm 2.2$ & $3 \pm 9$ & $17 \pm 17$ & $3.0 \pm 3.8$ & $63 \%(20 / 32)$ & $13 \%(4 / 32)$ & $38 \%(12 / 32)$ \\
\hline & $>0.42 \%$ & $\begin{array}{c}24 \% \\
(10 / 42)\end{array}$ & 7,3 & $13.3 \pm 3.2$ & $3.7 \pm 0.9$ & $1 \pm 1.6$ & $13 \pm 13$ & $2.5 \pm 3.1$ & $100 \%(10 / 10)$ & $0 \%(0 / 10)$ & $0 \%(0 / 10)$ \\
\hline
\end{tabular}

${ }^{\text {a } P C D A I}=$ Pediatric Crohn's Disease Activity Index

${ }^{\mathrm{b}} \mathrm{SED}=$ Erythrocyte sedimentation rate

${ }^{\mathrm{c}} 6$-Mercaptopurine, Azathioprine, Mesalamine, Prednisone

${ }^{d}$ Vedolizumab, Certolizumab, Ustekinumab, Infliximab-dyyb, Adalimumab

${ }^{\mathrm{e}}$ This one subject was only administered Remicade once.

${ }^{\mathrm{f}} \mathrm{NA}=$ Not applicable. 